\title{
The research of characteristics of the shear force transmission between the magneto-rheological fuid layer
}

\author{
Chunfu Gao, Guang Zhang ${ }^{\text {a }}$, Weizeng Chen, Xinsheng He, Lingyu Shi, Shihe Pan and Haiting Zhang \\ Institute of Machinery and Measurement-control Technology, Zhejiang Normal University, Jinhua 321004, China
}

\begin{abstract}
In order to studying the characteristics of MRF inter-layer shear force, through the development of MRF inter-layer shear force experiment device, under the action of dynamic composite field that composed of magnetic field and temperature field, the experiment analyzes the characteristics of magneto-rheological fluid force transmission between the layers and gain the inter-layer force characteristic curve, calculated the magneto-rheological fluid particles inter-atomic forces combined with the theoretical, and the results show that the wheel location characteristic curve of the MRF shear yield stressis symmetric axisym metric with wheel location, and the maximum shear yield stress on the axis of symmetry; Magneto-rheological fluid force characteristic curve moves between the layers mainly depends on coupling degree between the material properties of the experimental device and ferromagnetic particles of MRF. Keywords: magneto-rheological fluid; dynamic composite field; inter-layer force transmission; Shear.
\end{abstract}

\section{Introduction}

MRF[1] as a kind of smart material, its internal material is mainly composed of ferromagnetic particles, base load of fluid and additives [2, 3] that In order to prevent the ferromagnetic particles from unity and settlement. The transmission principle magneto-rheological Device is based on MRF as the working medium, the power and motion forms is to rely on two transmission interface between MRF to deliver, by adjusting the current size of the electromagnetic coil, to control the intensity of the Magneto-rheological effect, realize the MRF shear stress step-less adjustment.

Magneto-rheological transmission as a new form of power transmission, it not only has the characteristics of convenient control, low energy consumption, fast reaction (millisecond level), and the influence of the change of the external environment is very small. Therefore, magneto-rheological transmission technology is widely used in machine transmission parts of step-less speed regulation, the execution part of overload protection, mechanical and electrical equipment of soft start and soft brake, etc [4-7].

\footnotetext{
a Corresponding author : xzjx092@163.com
} 


\section{Experimental device}

Figure 1 shows the experimental device that study the characteristics of MRF inter-layer shear force.

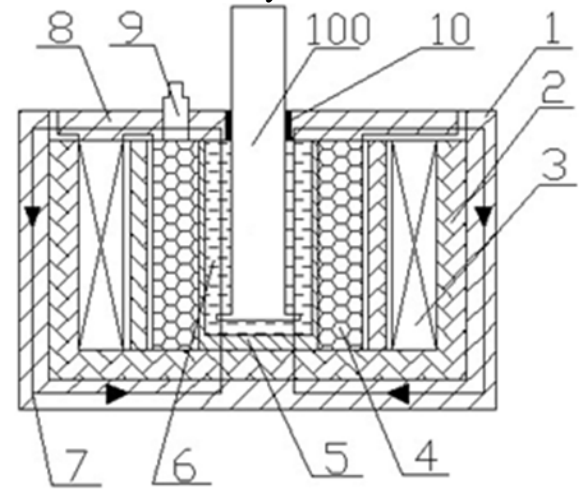

1.Round drum shell 2.Insulation pad 3.Electro magnetic coil 4.Aluminium heater 5.Aluminum alloy thin-walled cylinder 6.Magneto-rheological fluid 8.Tectum 9.Aluminium heater interface 10.Sealing shaft sleeve 100.The probe head

Figure 1. The experimental device that study the characteristics of MRF inter-layer shear force.
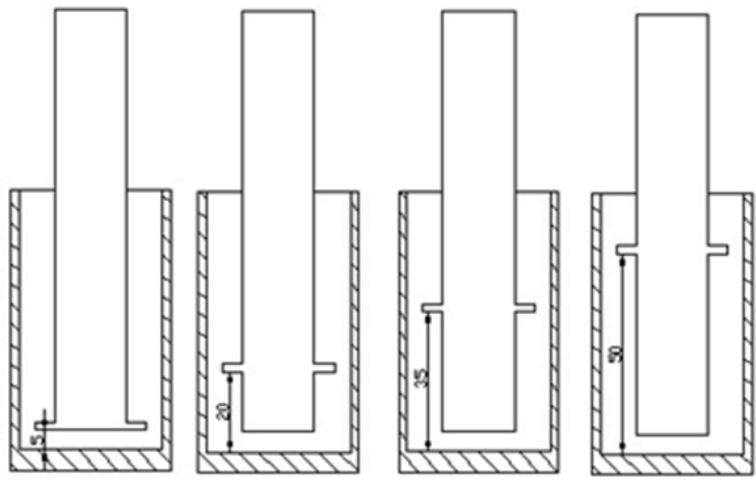

Figure 2. The four position of the detection head

By aluminium heater to evenly heating of MRF, controllable dynamic magnetic field was produced by the electromagnetic coil and the space full of MRF to form a dynamic composite field. Detection head was driven by motor to shear MRF, as shown in figure 2, shear experiments in different locations of MRF space was test by the fourprobe head.

\section{Theoretical analysis}

Under the action of shear force, the chain will be straight, but the distance between the ferromagnetic particlesin the same chain are far less than that between different chain corresponding to the distance of the ferromagnetic particles, therefore, local magnetization saturation effect and magnetic local accumulation effect can't be ignored when stress analysis in the same chain, so when suffer from the external shear force, the chain will be straight, assuming that the number of ferromagnetic particles in chain remains the same, the distance between ferromagnetic particles will change, because of the local magnetization saturation effect and magnetic local accumulation effect, make the chain of the inter-particle distance is not the same as the degree of change, leading to the force is different between the different position of ferromagnetic particles[8-9] of the whole chain, the stress analysis diagram as shown in figure 3. 


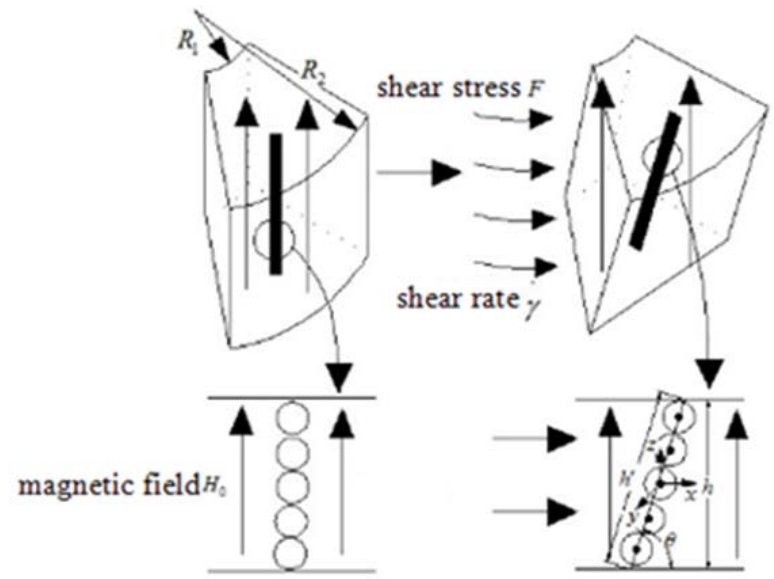

Figure 3. Force analysis of any $i$ ferromagnetic particles

In the figure 3, take any $i$ ferromagnetic particles for force analysis object, by the above analysis can get the interactions between the all magnetized ferromagnetic particles in MRF equivalent at the attraction between two objects that its core has a magnetic dipole moment of $m$. Assuming magnetic dipole moment of magnetized ferromagnetic particle of $i$ is $m_{i}$, and radius is $R_{i}$, on the basis of the provisions distance between magnetized ferromagnetic particleof $i$ and $i+1$ is $d_{i}$.

To force analysis between any magnetized ferromagnetic particleof $i-1$ and $i$. The magnetic dipole moment in their globe is:

$$
\begin{aligned}
& m_{i-1}=\frac{4 \pi \mu_{2}\left(\mu_{1}-\mu_{2}\right) R_{i-1}{ }^{3}}{2 \mu_{2}+\mu_{1}} H_{0}=-4 \pi \mu_{2} k_{2} R_{i-1}{ }^{3} H_{0} \\
& m_{i}=\frac{4 \pi \mu_{2}\left(\mu_{1}-\mu_{2}\right) R_{i}^{3}}{2 \mu_{2}+\mu_{1}} H_{0}=-4 \pi \mu_{2} k_{2} R_{i}^{3} H_{0}
\end{aligned}
$$

The acting force between them can be represented as:

$$
\begin{aligned}
& \vec{\tau}_{i(i-1)}^{\prime}=\frac{m_{i-1} m_{i}}{4 \pi \mu_{0} d_{i-1}^{2}} \vec{e}_{i-1} \\
& \vec{\tau}_{(i-1) i}^{\prime}=\frac{m_{i-1} m_{i}}{4 \pi \mu_{0} d_{i-1}^{2}} \vec{e}_{i}
\end{aligned}
$$

Rules that $\vec{\tau}_{i(i-1)}^{\prime}$ was expressed as $i-1$ force on $i, \vec{e}_{i-1}$ was expressed as direction from the a particle $i$ to the particles $i-1$. As a result, they are a pair of action and reaction (of the same size in the opposite direction).

To force analysis between any magnetized ferromagnetic particleof $i$ and $i+1$, the magnetic dipole moment in their globe are:

$$
m_{i+1}=\frac{4 \pi \mu_{2}\left(\mu_{1}-\mu_{2}\right) R_{i+1}{ }^{3}}{2 \mu_{2}+\mu_{1}} H_{0}=-4 \pi \mu_{2} k_{2} R_{i+1}{ }^{3} H_{0}
$$


The acting force between them can be represented as:

$$
\begin{gathered}
\vec{\tau}_{i(i+1)}^{\prime}=\frac{m_{i+1} m_{i}}{4 \pi \mu_{0} d_{i}^{2}} \vec{e}_{i+1} \\
\vec{\tau}_{(i+1) i}^{\prime}=\frac{m_{i+1} m_{i}}{4 \pi \mu_{0} d_{i}^{2}} \vec{e}_{i}
\end{gathered}
$$

As a result, they are for a pair of action and reaction (same size in the opposite direction).

\section{Experiments and results analysis}

Figure 4, figure 5 and figure 6 respectively are given under the condition of shear rate of $\gamma=30 S^{-1}$, temperature of $T=25^{\circ} \mathrm{C}$, when the magnetic induction intensity of $B=50 \mathrm{mT}, 110 \mathrm{mT}, 170 \mathrm{mT}, 215 \mathrm{mT}$, the shear rate of $\gamma=30 S^{-1}, 114 S^{-1}, 196 S^{-1}, 263 S^{-1}$, the temperature of $T=25^{\circ} \mathrm{C}, 75^{\circ} \mathrm{C}, 125^{\circ} \mathrm{C}, 160^{\circ} \mathrm{C}$, the relationship between MRF shear yield stress and the location of the rotary table.

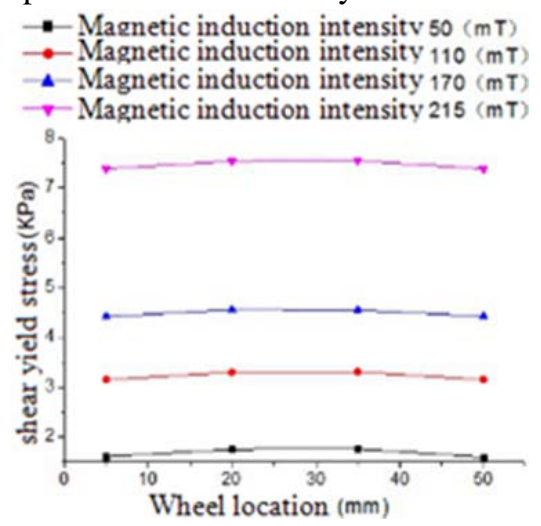

Figure 4. Magnetic induction intensity changes

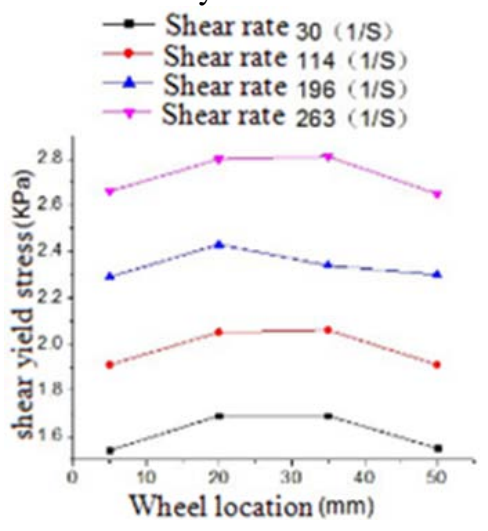

Figure 5. shear rate changes

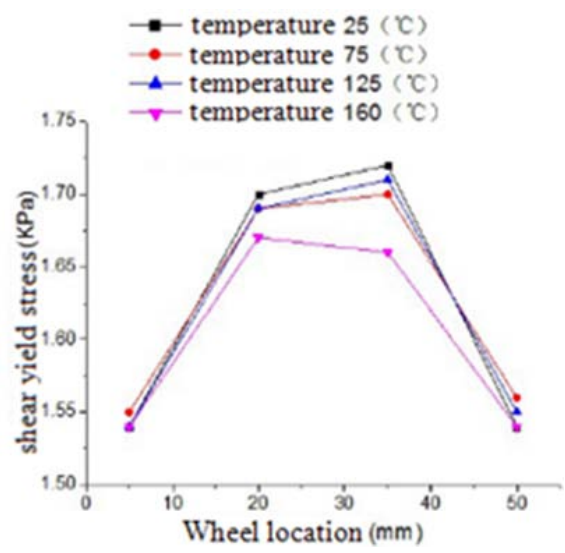

Figure 6. Temperature change

Combined with figure 4 and figure 5 and figure 6 MRF shear yield stress of the wheel location characteristic curve shows that the curve is symmetric axis-ymmetric with wheel location at $h=27.5 \mathrm{~mm}$, and the maximum shear yield stress on the axis of symmetry, in the magnetic induction 
intensity for $215 \mathrm{mt}$, the shear yield stress can reach $7.5 \mathrm{Kpa}$.

Respectively compared the rotary wheel location at $h=20 \mathrm{~mm}$ with $h=5 \mathrm{~mm}$ of the magneto-rheological fluid layer, shear yield stress relative increment are $0.14 m T, 0.15 m T, 0.14 m T, 0.15 m T$ and $0.15 m T, 0.14 m T, 0.14 m T, 0.14 m T$ respectively, is almost equal increment between them, visible known that in the MRF ferromagnetic particles magnetized under the condition of uniform, Magneto-rheological fluid force characteristic curve moves between the layers mainly depends on coupling degree between the material properties of the experimental device and ferromagnetic particles of MRF.

\section{Summary}

In this paper, through establishing MRF inter-layer shear properties research device, by the dynamic field that composite magnetic field and temperature field, and experiment research the different position of MRF in space respectively, and combining with theoretical analysis, inter-layer particles force calculation model is set up, the conclusion is as follows:

(1) The curve is symmetric axis-ymmetric with wheel location at $h=27.5 \mathrm{~mm}$, and the maximum shear yield stress on the axis of symmetry, in the magnetic induction intensity for $215 \mathrm{mt}$, the shear yield stress can reach $7.5 \mathrm{Kpa}$.

(2) In the MRF ferromagnetic particles magnetized under the condition of uniform, Magneto-rheological fluid force characteristic curve moves between the layers mainly depends on coupling degree between the material properties of the experimental device and ferromagnetic particles of MRF.

(3) The relationship between Magneto-rheological fluid force characteristic curve moves between the layers and the magnetic induction intensity and the relationship between shear rate is not close, they just changed the whole curve in the shear yield stress on the direction of movement.

\section{Acknowledgements}

Foundation item: The national natural science foundation of China (51275483); Nature Science Fund of Zhejiang province (LY13E050014).

\section{References}

1. Carlson J D. Magnetorheological fluid actuators [A]. Janocha H. Adaptronics and smart structures[C]. Berlin: Springer-verlag Berlin Heidelberg, 180-195(1999).

2. Ginder J M. Behavior of magnetorheological fluids [J]. MRS Bulletin of Applied Physics, 20: 1137-1140(1949).

3. Dai S, Du C, Yu G. Design, testing and analysis of a novel composite magnetorheological fluid clutch[J]. Journal of intelligent Material Systems and Structures, 24(14): 1675-1682(2013).

4. Milecki A Investigation and control of magnrtorheological fluid dampers [J]. International Journal of Machine Tools and Manufacture, 41(3): 379-391(2001).

5. Meng Q R, Hou Y F. Mechanism of hydro-viscous soft start of belt conveyor [J]. Journal of China University of Mining and Technology, 18(3): 459-465( 2008).

6. Hayat T, Nadeem S, Asghar S. Periodic Unidirectional Flows of a Viscoelastic Fluid with the Fractional Maxwell Mode[J]. Applied Mathematics and Computation, 151(1): 153-161, (2004).

7. Rabinow J. The magnetic fluid clutch [J]. AIEE Transactions, 1948, 67: 1308-1315.

8. Carlson J D, Catanzarite D M, Clair K A. Commercial magnetorheological fluid devices [J]. Internetional Journal of Modern Physics B. 10(23-24): 2857-2865(1996).

9. Bossis G, Volkova O, Lacis S and Meunier A, Magnetorheology: fluids, structures andrheology[J]. Lecture Notes in Physics, 594: 202-230(2003). 